\title{
Synthesis and Thermal Stability of B20-Type TMGe (TM = Mn, Fe and Co) Intermetallic Compounds Prepared by Mechanical Milling
}

\author{
Takanobu Hiroto $^{1, *}$, Yeong-Gi $\mathrm{So}^{2}$ and Kaoru Kimura ${ }^{1}$ \\ ${ }^{1}$ Graduate School of Frontier Sciences, The University of Tokyo, Kashiwa 277-8561, Japan \\ ${ }^{2}$ Department of Materials Science, Akita University, Akita 010-8502, Japan
}

We propose a new synthesis method of transition-metal monogermanides (TMGe, TM = Mn, Fe, and Co) with a B20-type chiral structure. Arc-melting followed by mechanical milling can be used to produce 70-110 A-sized B20-type structures. Differential scanning calorimetry and heat-treatment tests indicated that the B20-phases are stable below $673 \mathrm{~K}, 873 \mathrm{~K}$ and $773 \mathrm{~K}$ for MnGe, FeGe and CoGe, respectively. We also demonstrated that sintering tests are able to yield dense compacts from the FeGe and CoGe B20-phase powders. [doi:10.2320/matertrans.M2018016]

(Received January 12, 2018; Accepted March 19, 2018; Published April 20, 2018)

Keywords: B20-type transition-metal monogermanide, X-ray diffraction, mechanical milling, spark plasma sintering

\section{Introduction}

Transition-metal monogermanides (TMGe, $\mathrm{TM}=\mathrm{Cr}, \mathrm{Mn}$, $\mathrm{Fe}, \mathrm{Co}$, and $\mathrm{Ru}$ ) with a B20-type chiral structure (Fig. 1(a)) exhibit various physical properties, from semiconducting $\mathrm{RuGe}^{1)}$ to metallic helical magnetic FeGe. ${ }^{2)}$ B20-type intermetallic compounds are of recent interest for two reasons, namely, for the formation of magnetic skyrmions ${ }^{3-8)}$ and in thermoelectric material applications. ${ }^{11)}$

Magnetic skyrmions, which are defined as topologically protected vortex-like spin objects, appear as non-centrosymmetric chiral magnets, such as $\mathrm{MnSi}^{3,4}{ }^{3,}\left(\mathrm{Fe}_{1-x} \mathrm{Co}_{x}\right) \mathrm{Si}^{5,6}$ FeGe, $\left.{ }^{7}\right)\left(\mathrm{Mn}_{1-x} \mathrm{Fe}_{x}\right) \mathrm{Ge},{ }^{8)}$ and recently discovered $\mathrm{Co}-\mathrm{Zn}-$ Mn compounds. ${ }^{9)}$ The former monosilicides and monogermanides belong to the same non-centrosymmetric space group of $P 2{ }_{1} 3$, and are a B20-type structure, whereas the latter $\mathrm{Co}-\mathrm{Zn}-\mathrm{Mn}$ compounds have a $P 4_{1} 32$ or $P 4_{3} 32$, which depends on their handedness, and their crystal structure is isostructural to the $\beta$ - $\mathrm{Mn}$ structure. The spatial inversion symmetry breaking is an essentially important in the formation of skyrmions, and most studies on magnetic skyrmions in metallic system have focused on the B20type compounds. ${ }^{10)}$ The FeGe B20-phase has the highest transition temperature of $T_{\mathrm{c}} \sim 278 \mathrm{~K}$ (almost near room temperature) among the B20-type chiral magnets, ${ }^{7)}$ which may indicate that it is a good candidate for application in skyrmions for next-generation magnetic memory devices.

With regards the transport properties of the TMGe B20 compounds, a larger negative thermoelectric power of $-82 \mu \mathrm{V} / \mathrm{K}$ (at room temperature) is reported because of an asymmetric band with a pseudo-gap electronic structure in CoGe, and a resultant higher dimensionless figure of merit $(Z T=\sim 0.11)$ among the other B20-type compounds. ${ }^{11)} Z T$ is defined as:

$$
Z T=\frac{S^{2} \sigma}{\kappa} T
$$

*Present address: National Institute for Materials Science, Tsukuba 305 0047, Japan. Corresponding author, E-mail: HIROTO.Takanobu@nims. go.jp where $S, \sigma, \kappa$, and $T$ are the Seebeck coefficient, electrical conductivity, thermal conductivity, and absolute temperature, respectively. Although this $Z T$ value is low for commercial use $(Z T \sim 1)$, the power factor $\left(S^{2} \sigma=\sim 3 \mathrm{~mW} / \mathrm{mK}^{2}\right)$ is as comparably high as commercially used $\mathrm{Bi}-\mathrm{Te}$-based thermoelectric materials $\left(5.5 \mathrm{~mW} / \mathrm{mK}^{2}\right.$ for the p-type and $3.3-$ $4.3 \mathrm{~mW} / \mathrm{mK}^{2}$ for the n-type) at $\sim 300 \mathrm{~K} .{ }^{12)}$ The main reason for a suppressed $Z T$ value in CoGe results from the relatively large thermal conductivity component $(\sim 8.9 \mathrm{~W} / \mathrm{mK}$ at $300 \mathrm{~K})$. Therefore, if a reduction in lattice thermal conductivity by nanostructuring is achieved, the $Z T$ values should be enhanced further. Theoreticians predict a new mechanism for the enhancement of thermoelectric power (or Nernst effect) in the presence of magnetic skyrmions. ${ }^{13)}$ In other words, a series of TMGe compounds has significant potential application in advanced magnetic and/or thermoelectric materials.

Tiny single crystals $(\sim 1 \mathrm{~mm})$ have been prepared via the vapor transport method, ${ }^{14)}$ and polycrystalline TMGe B20-type compounds have typically been obtained by highpressure and high-temperature (HPHT) synthesis at $\sim 4-$ $5 \mathrm{GPa}$ and $\sim 1000 \mathrm{~K} .^{15)}$ The limited sample volume of the high-pressure apparatus is unsuitable for the large-scale production of B20-type compounds. Thus, an easy largescale synthesis of the B20-type TMGe compound series is required. In previous reports, the B20-type FeGe phase has been produced by mechanical-milling from raw-material powder. ${ }^{16,17)}$ The formation and/or thermal stability of other binary TMGe B20-phases has not been reported.

We synthesized a series of TMGe (TM = Mn, Fe, and Co) B20-type powders by mechanical milling, and discuss and characterize the thermal stability of the obtained powders. We also prepared sintered compacts by using the spark plasma sintering (SPS) method. Our method is a convenient alternative to HPHT synthesis for preparing B20-type TMGe compounds and may be useful for investigations of magnetic skyrmions and thermoelectric materials.

\section{Experimental Method}

Stoichiometric amounts of high-purity (>99.9 mass\% 
(a)

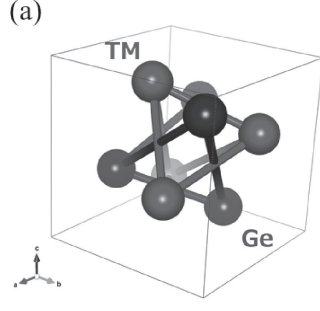

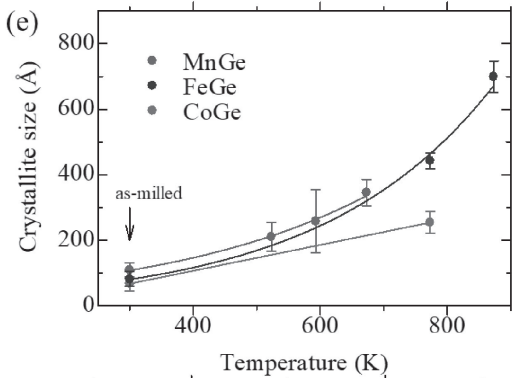

(b)

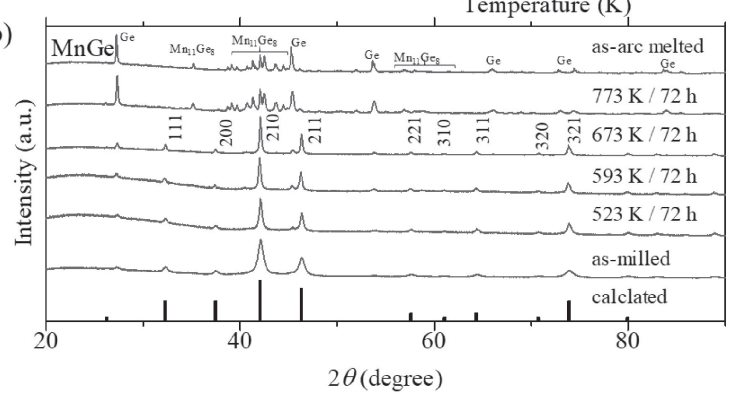

(c)

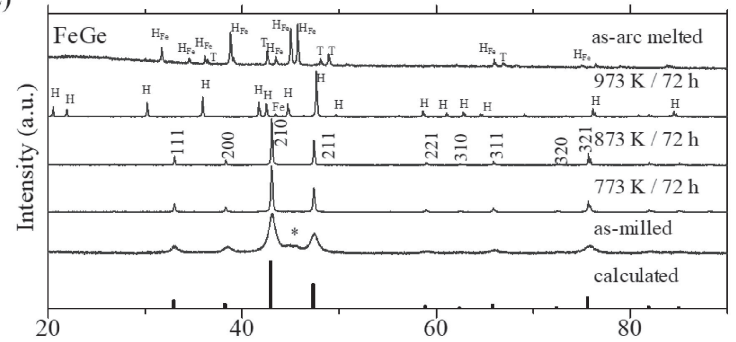

(d)

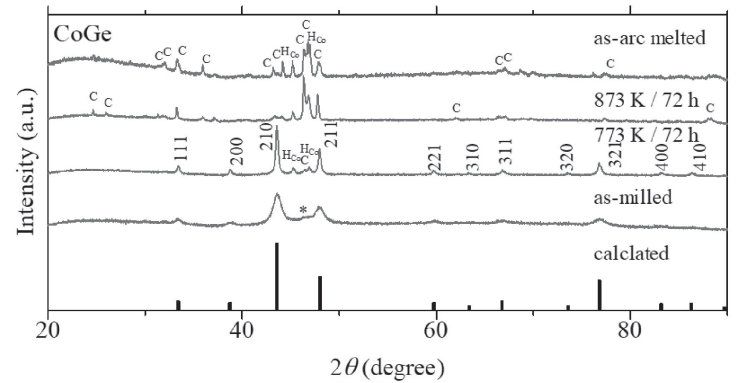

Fig. 1 (a) The crystal structure of the TMGe (TM = Mn, Fe and Co) B20phase was constructed by using the VESTA 3 program package. ${ }^{25)}$ (b)-(d) Powder XRD patterns of investigated TMGe $(\mathrm{TM}=\mathrm{Mn}, \mathrm{Fe}$, and $\mathrm{Co})$ compounds. The lower vertical bars in each figure represents the calculated intensity distribution of the TMGe B20-phase. The upper part shows the as-milled and annealed powder sample at appropriate temperatures for $72 \mathrm{~h}$. For comparison, samples after arc-melting are shown in the top part. The notation $\mathrm{Ge}, \mathrm{Mn}_{11} \mathrm{Ge}_{8}, \mathrm{H}_{\mathrm{Fe}}, \mathrm{T}, \mathrm{Fe}, \mathrm{H}$, and $\mathrm{C}$ represents the identified phases. $\mathrm{H}_{\mathrm{Fe}}, \mathrm{H}, \mathrm{C}$, and $\mathrm{H}_{\mathrm{Co}}$ are the Fe-rich hexagonal- $\mathrm{Fe}_{13} \mathrm{Ge}_{8}$, high-temperature hexagonal $\mathrm{FeGe}$ (or $\mathrm{CoGe}$ ) and $\mathrm{C}$ centered-monoclinic-CoGe, and Co-rich hexagonal $\mathrm{Co}_{5} \mathrm{Ge}_{3}$ respectively. Asterisks are unidentified phases. (e) The annealing temperature dependence of the crystallite sizes of the B20-phases is estimated by the Scherrer method. Solid lines are guides for the eye.

purity) elemental $\mathrm{Mn}, \mathrm{Fe}, \mathrm{Co}$, and $\mathrm{Ge}$ were weighed as $\mathrm{TM}: \mathrm{Ge}=1: 1$ and melted by an arc-melting method on a water-cooled $\mathrm{Cu}$ hearth (NEV-AD-03 or NEV-ACD-05; Nissin-Giken Co., Ltd., Saitama, Japan). Mother ingots were reduced to small pieces, and fine powders were obtained by using a tilted planetary ball-mill (Planet Min; Nagao system Inc., Kanagawa, Japan) with a zirconia jar and balls (5-mm diameter) at a rotor speed of $600 \mathrm{rpm}$ for $1 \mathrm{~h}$. The milling conditions are provided in Ref. 18). The mass ratio of sample and balls was set as $3 \mathrm{~g}: 80 \mathrm{~g}$. The sample phase characterization was performed by X-ray diffractometry (XRD) using $\mathrm{Cu} K \alpha$ radiation with an operating voltage and current of $40 \mathrm{kV}$ and $30 \mathrm{~mA}$, respectively (SmartLab; Rigaku Co., Ltd., Tokyo, Japan). The thermal stability of the as-milled powders was determined by differential scanning calorimetry (DSC, DSC7020; Hitachi High-Technologies Co., Ltd., Tokyo, Japan) and by heat treatment at various temperatures for $72 \mathrm{~h}$ in an Ar atmosphere. The ramp-up rate for the DSC measurement was chosen as $10 \mathrm{~K} / \mathrm{min}$. The maximum temperature of the DSC measurement was $873 \mathrm{~K}$.

Based on the thermal stability experiments, the sintering test for FeGe and CoGe was performed by SPS to form a bulk sample. In the SPS process, we chose the sintering pressure and temperature as $600 \mathrm{MPa}$ and $773 \mathrm{~K}$, respectively, to obtain a high-density sample. The holding time at $773 \mathrm{~K}$ was set to $10 \mathrm{~min}$. Sintered compacts were annealed at $873 \mathrm{~K}$ for $\mathrm{FeGe}$ or at $773 \mathrm{~K}$ for $\mathrm{CoGe}$ for $72 \mathrm{~h}$ in Ar. Bulk sample densities were measured by the Archimedes method using water. The microstructures were studied by scanning electron microscopy (SEM) with an operating voltage of $20 \mathrm{kV}$ (JSM6010LA, JEOL Co., Ltd., Tokyo, Japan).

\section{Results and Discussion}

Figures 1(b), (c), and (d) show powder XRD patterns for (b) MnGe, (c) FeGe, and (d) CoGe for the as-milled and annealed samples at several temperatures for $72 \mathrm{~h}$. All main peaks of the as-milled samples, except for small broad peaks notated by asterisks, can be indexed as a cubic lattice with $a=4.796(5) \quad(\mathrm{MnGe}), 4.696(3) \quad(\mathrm{FeGe})$, and 4.646(4) $\AA$ (CoGe), which is consistent with the reported B20-type compounds of $a=4.7925(\mathrm{MnGe}){ }^{15)} 4.6995(2) \AA(\mathrm{FeGe}),{ }^{19)}$ and $4.637(3) \AA(\mathrm{CoGe}),{ }^{20)}$ respectively. The intensity distributions are also similar to those of the calculated distributions $^{15,19,20)}$ (as shown by the vertical bars). Thus, we conclude the successful synthesis of the series of TMGe with a B20-structure by ball-milling within $1 \mathrm{~h}$. The salient broadness of the Bragg peaks of the as-milled samples can be explained by the crystallite size effect as addressed later. No B20-phases formed after the arc-melted sample (see each upper figure), i.e., $\mathrm{Mn}_{11} \mathrm{Ge}_{8}+\mathrm{Ge}, \mathrm{H}_{\mathrm{Fe}}$ (Hexagonal Ferich $\left.\mathrm{Fe}_{13} \mathrm{Ge}_{8}\right)+\mathrm{T}$ (Tetragonal $\left.\mathrm{FeGe}_{2}\right)$, and $\mathrm{C}(C$-centeredmonoclinic $\mathrm{CoGe})+\mathrm{H}_{\mathrm{Co}}$ (Hexagonal Co-rich $\mathrm{Co}_{5} \mathrm{Ge}_{3}$ ) phases exist for $\mathrm{MnGe}$, FeGe, and $\mathrm{CoGe}$ mother ingots, respectively. As a result, the mechanical-milling process is critically important for the formation of B20-type phases. We do not discuss the detailed formation mechanism of the B20-phases during mechanical milling; however, a local melting mechanism is proposed in the FeGe case. ${ }^{15)}$ Here, asterisks in Fig. 1(c) and (d) is should to be assigned as the intermediate phase toward B20-phase and/or nano-sized $\mathrm{H}_{\mathrm{Fe}}, \mathrm{H}_{\mathrm{Co}}$ and $\mathrm{C}$ phase.

To discuss the thermal stability of the synthesized B20phases, we measured the DSC curve up to $873 \mathrm{~K}$ for each sample as shown in Fig. 2. An endothermic peak was only observed at approximately $755 \mathrm{~K}$ for $\mathrm{MnGe}$, whereas, exothermic peaks were observed at approximately $560 \mathrm{~K}$ and $620 \mathrm{~K}$ for $\mathrm{MnGe}, 640 \mathrm{~K}$ for $\mathrm{FeGe}$, and $640 \mathrm{~K}$ for CoGe. Based on the DSC measurement and the later thermal- 


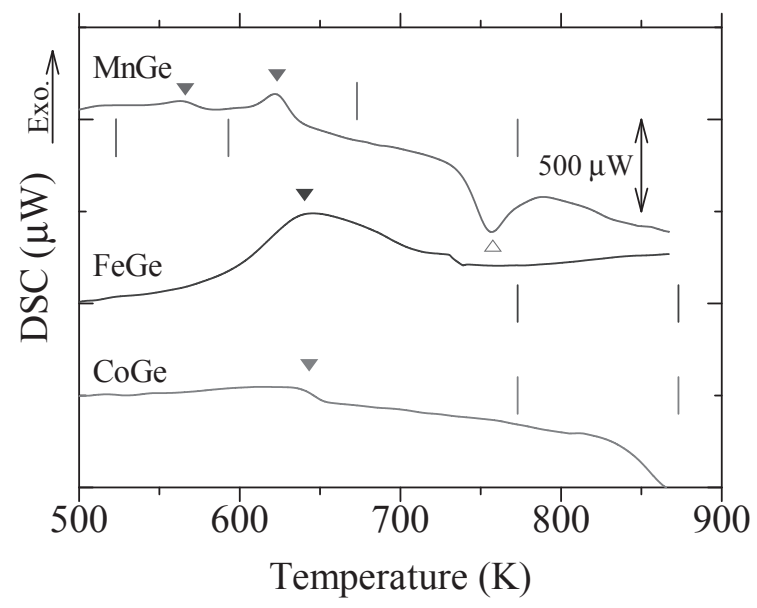

Fig. 2 DSC curves for MnGe, FeGe, and CoGe. The ramp-up rate is set to $10 \mathrm{~K} / \mathrm{min}$. Inverted filled triangles and open triangles represent the exothermic and endothermic peaks, respectively. Vertical bars show the heat-treatment temperatures that we tested for $72 \mathrm{~h}$ (see also Fig. 1).

treatment test (Figs. 1(b)-(d)), the endothermic peak corresponds to a decomposition of the B20-phase. No significant difference in phases was observed between the exothermic peaks, which may result from the sintering or grain growth effect to reduce the surface energy of the nanosized particle.

We used heat-treatment tests combined with DSC data to study the temperature stability of the B20-phases. As shown in Figs. 1(b)-(d), upon heating, the Bragg peaks become increasingly sharper and finally, the B20-phase decomposes to $\mathrm{Mn}_{11} \mathrm{Ge}_{4}+\mathrm{Ge}$, FeGe (hexagonal phase) + bcc-Fe, and $\mathrm{CoGe}(C$-centered-monoclinic phase) for $\mathrm{MnGe}, \mathrm{FeGe}$, and CoGe, respectively.

Figure 1(e) shows the crystallite size for each temperature process, as estimated from the integral breadth of the Bragg peak, $\beta$, by using the conventional Scherrer equation:

$$
D=\frac{K \lambda}{\beta \cos \theta},
$$

where $D, \lambda$, and $\theta$ are the crystallite size, the X-ray wavelength, and the Bragg angle, respectively. $K$ is the shape factor (Scherrer constant) and we chose $K=1$ in this study. Each mean crystallite size $D$ is estimated from some Bragg peaks. The peak-broadening from the lattice-strain effect during ball milling is negligible because no significant evidence of lattice strain exists in the Williamson-Hall plot ${ }^{21)}$ compared with the Si standard (NIST 640d). The crystallite sizes for the as-milled $\mathrm{MnGe}, \mathrm{FeGe}$, and $\mathrm{CoGe}$ powders were estimated as $110 \pm 20,80 \pm 20$, and $70 \pm 20 \AA$, respectively. Upon heating, before the decomposition of the B20-phase, the crystallite grows to $350 \pm 40,700 \pm 50$, and $250 \pm 50 \AA$ for $\mathrm{MnGe}, \mathrm{FeGe}$, and $\mathrm{CoGe}$, respectively. Note that the measurable crystallite size is estimated to be less than $1000 \AA$ under our experimental conditions.

The crystallite size of the FeGe increases significantly with an increase in annealing temperature. Such increases are not observed clearly for the MnGe and CoGe powders under our heat-treatment conditions. The difference may result from their thermal stability; according to the TMGe binary phase diagrams, ${ }^{22,23)}$ only the FeGe B20-phase is thermodynamically stable up to $903 \mathrm{~K}$, i.e., stable $\mathrm{MnGe}$ and CoGe
B20-phases are not reported at ambient pressure. Therefore, our method can produce metastable B20-phases at ambient pressure without a HPHT synthesis. Note that our method is also efficient to prepare the B20-type solid-solutions, such as the $\left(\mathrm{Fe}_{1-x}, \mathrm{Mn}_{x}\right) \mathrm{Ge}$ compounds, ${ }^{26)}$ which have thus far been synthesized only by a HPHT technique. ${ }^{8)}$

The estimated crystallite size for FeGe is comparable with or smaller than the reported magnetic skyrmion size of $\left.\sim 700 \AA .{ }^{7}\right)$ Thus, the formation of the magnetic skyrmions in our samples may be important and our approach may provide insight into the formation of magnetic skyrmions over that obtained from epitaxial thin-film studies. ${ }^{24)}$

It may be necessary to apply TMGe compounds in bulk, so we performed sintering experiments to obtain a FeGe and CoGe bulk sample. We also attempted the SPS method on MnGe powder; however, we failed to obtain sintering compacts because of the relatively low decomposition temperature $(<773 \mathrm{~K})$ of the MnGe B20-phase (see Fig. 1(a)). Figure 3(a) and (b) show SEM images of sintered FeGe and CoGe, respectively. Besides the small pore size $(<\sim 50 \mu \mathrm{m})$ in the CoGe sample, no significant cracks were observed. The measured bulk densities are 7.92 and $8.13 \mathrm{~g} / \mathrm{cm}^{3}$, and the relative density from a structural model of the B20-phase is $96.3 \%$ and $92.4 \%$ for FeGe and CoGe, respectively. Figure 2(c) shows the XRD patterns of sintered compacts of FeGe and CoGe. Except for small amounts of impurities denoted by asterisks in CoGe, all peaks can be assigned as a B20-phase. Thus, the sintering method is able to yield dense compacts from our powder. The thermal conductivity for sintered compacts of CoGe is $6.3 \mathrm{~W} / \mathrm{mK}$ at $377 \mathrm{~K}$, which is much lower than the literature value $\left(8.9 \mathrm{~W} / \mathrm{mK}\right.$ at $\left.300 \mathrm{~K}^{11)}\right)$. Thus, our method is efficient for about $30 \%$ reduction of the thermal conductivity. The main reason for that is from an increase in the phonon scattering effect by the nanocrystalline structure. Whereas, the obtained $Z T$ value is low as 0.053 at $373 \mathrm{~K}$, which is about half of literature $(Z T \sim 0.11$ at $300 \mathrm{~K}) .{ }^{11)}$ The suppressed $Z T$ value is understood by the relatively small Seebeck coefficient $\left(-45 \mu \mathrm{V} / \mathrm{K}\right.$ at $373 \mathrm{~K} ;-82 \mu \mathrm{V} / \mathrm{K}$ at $300 \mathrm{~K}$ in literature $\left.{ }^{11)}\right)$ in our sample, possibly due to the influence of secondly phases. Further improvement of $Z T$ in the transitionmetal monogermanide B20-phases should be achieved by optimizing the synthesis conditions.

\section{Conclusions}

We propose a new convenient synthesis method to prepare TMGe $(\mathrm{TM}=\mathrm{Mn}, \mathrm{Fe}$, and Co) with a B20-type chiral structure. B20-type TMGe compounds with the crystallite size of $70-110 \AA$ were synthesized by mechanical milling within $1 \mathrm{~h}$ after arc-melting. The crystallite size of B20phases increased by heat treatment, and which finally decomposed into other crystalline phases over $673 \mathrm{~K}$, $873 \mathrm{~K}$, and $773 \mathrm{~K}$ for $\mathrm{MnGe}, \mathrm{FeGe}$, and CoGe, respectively. We also performed sintering experiments on the FeGe and CoGe B20-phases by SPS method, and successfully obtained a high-density bulk form. The thermal conductivity for sintered compacts of CoGe efficiently decreased by $30 \%$ compared with that for CoGe synthesized by a HPHT process. Our method is suitable for large-scale synthesis 


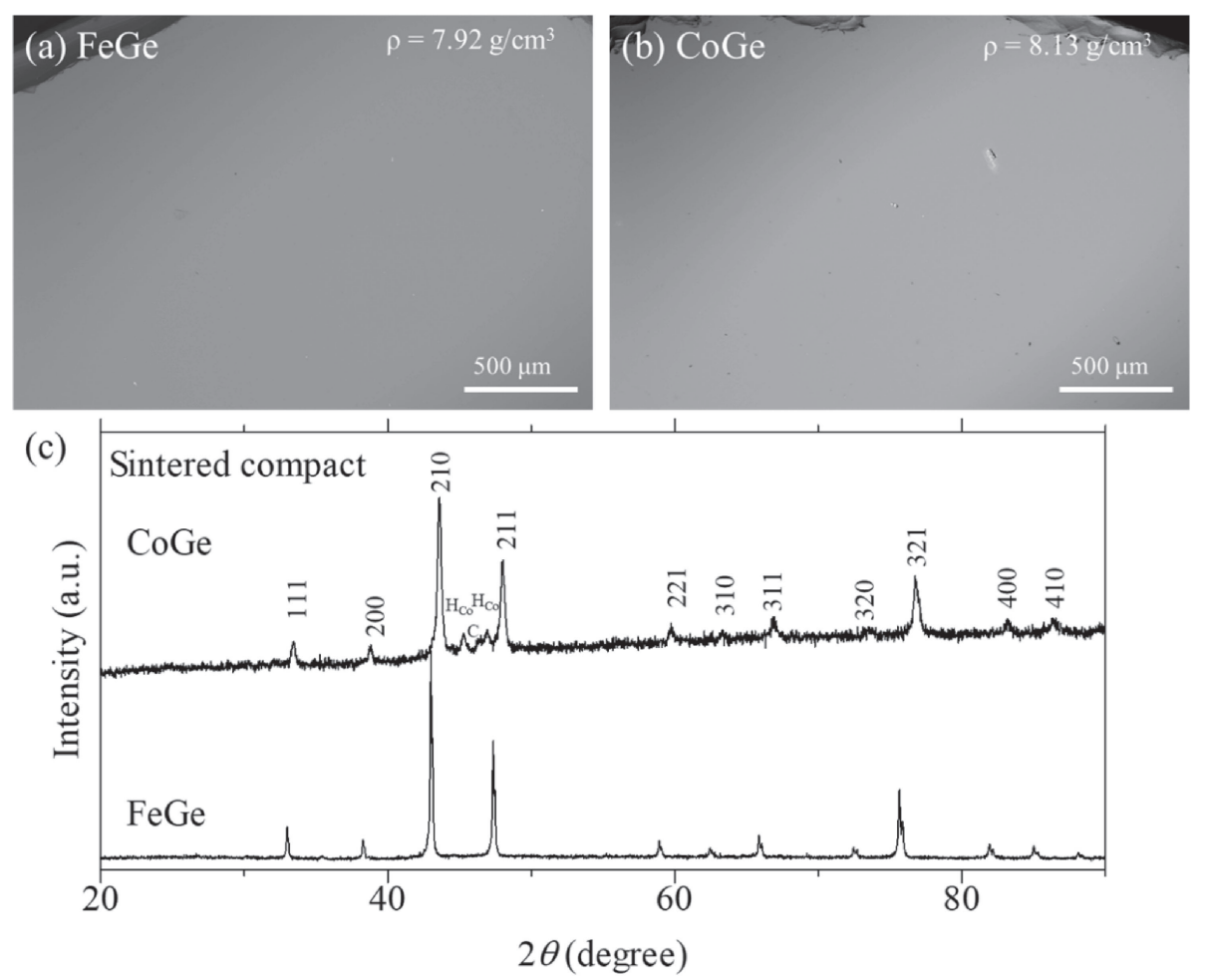

Fig. 3 Backscattered electron microscope image of (a) FeGe and (b) CoGe. The measured sample bulk density is also shown. Besides the sub-100- $\mu \mathrm{m}$ pores as shown in (b), no apparent cracks are shown in both samples. (c) XRD patterns of sintered FeGe and CoGe. Besides the small amounts of impurity phases denoted as $\mathrm{H}_{\mathrm{Co}}$ and $\mathrm{C}$ (same notation as Fig. 1) in CoGe, all peaks can be assigned as a B20-phase.

of B20-type transition-metal monogermanides aiming to the applied research of magnetic skyrmions and/or highly efficient thermoelectric materials.

\section{Acknowledgments}

T. H. acknowledges a Grant-in-Aid for JSPS fellows (15J04682) from the Ministry of Education, Culture, Sports, Science and Technology of Japan. We thank Edanz Group (www.edanzediting.com/ac) for editing a draft of this manuscript.

\section{REFERENCES}

1) H. Hohl, A.P. Ramirez, C. Goldman, G. Ernst and E. Bucher: J. Alloys Compd. 278 (1998) 39.

2) B. Lebech, J. Bernhard and T. Freltoft: J. Phys. Condens. Matter 1 (1989) 6105

3) S. Mühlbauer, B. Binz, F. Jonietz, C. Pfleiderer, A. Rosch, A. Neubauer, R. Georgii and P. Böni: Science 323 (2009) 915.

4) A. Tonomura, X.Z. Yu, K. Yanagisawa, T. Matsuda, Y. Onose, N. Kanazawa, H.S. Park and Y. Tokura: Nano Lett. 12 (2012) 1673.

5) W. Münzer, A. Neubauer, T. Adams, S. Mühlbauer, C. Franz, F. Jonietz, R. Georgii, P. Böni, B. Pedersen, M. Schmidt, A. Rosch and C. Pfleiderer: Phys. Rev. B 81 (2010) 041203(R).

6) X.Z. Yu, Y. Onose, N. Kanazawa, J.H. Parc, J.H. Han, Y. Matsui, N. Nagaosa and Y. Tokura: Nature 465 (2010) 901.

7) X.Z. Yu, N. Kanazawa, Y. Onose, K. Kimoto, W.Z. Zhang, S. Ishiwata, Y. Matsui and Y. Tokura: Nat. Mater. 10 (2011) 106.
8) S.V. Grigoriev, N.M. Potapova, S.-A. Siegfried, V.A. Dyadkin, E.V. Moskvin, V. Dmitriev, D. Menzel, C.D. Dewhurst, D. Chernyshov, R.A. Sadykov, L.N. Fomicheva and A.V. Tsvyashchenko: Phys. Rev. Lett. 110 (2013) 207201.

9) Y. Tokunaga, X.Z. Yu, J.S. White, H.M. Rønnow, D. Morikawa, Y. Taguchi and Y. Tokura: Nat. Commun. 6 (2015) 7638.

10) Seki and M. Mochizuki: Skyrmions in Magnetic Materials, (SpringerBriefs in Physics, 2015) chapter 2.

11) N. Kanazawa, Y. Onose, Y. Shiomi, S. Ishiwata and Y. Tokura: Appl. Phys. Lett. 100 (2012) 093902.

12) O. Yamashita and S. Tomiyoshi: J. Appl. Phys. 93 (2003) 368.

13) Y.P. Mizuta and F. Ishii: Sci. Rep. 6 (2016) 28076.

14) M. Richardson: Acta Chem. Scand. 21 (1967) 2305.

15) H. Takizawa, T. Sato, T. Endo and M. Shimoda: J. Solid State Chem. 73 (1988) 40.

16) Y.S. Kwon, K.B. Gerasimov, O.I. Lomovsky and S.V. Pavlov: J. Alloys and Compd. 352 (2003) 194.

17) I.A. Sviridov, L.M. Kubalova, O.Ya. Vasil'eva and V.I. Fadeeva: Inorg. Mater. 42 (2006) 867.

18) N. Sato, H. Ouchi, Y. Takagiwa and K. Kimura: Chem. Mater. 28 (2016) 529.

19) H. Wilhelm, M. Schmidt, R. Cardoso-Gil, U. Burkhardt, M. Hanfland, U. Schwarz and L. Akselrud: Sci. Technol. Adv. Mater. 8 (2007) 416.

20) V.I. Larchev and S.V. Popova: J. Less Common Met. 87 (1982) 53.

21) G.K. Williamson and W.H. Hall: Acta Metall. 1 (1953) 22.

22) K.B. Gerasimov and S.V. Pavlo: Intermetallics 8 (2000) 451.

23) See Landolt-Börnstein datasheet, by B. Predel (edited by O. Madelung), (Springer, 1996) and references therein.

24) S.X. Huang and C.L. Chien: Phys. Rev. Lett. 108 (2012) 267201.

25) K. Momma and F. Izumi: J. Appl. Cryst. 44 (2011) 1272.

26) T. Hiroto, T.J. Sato, Y.-G. So and K. Kimura: (unpublished). 